\title{
Heterogeneous Droplet Catalyst for Selective Oxidation of Methane
}

\section{Yuen Wu ( $\nabla$ yuenwu@ustc.edu.cn )}

University of Science and Technology of China https://orcid.org/0000-0001-9524-2843

\section{Haoran Zhang}

University of Science and Technology of China

\section{Xiaoqian Wang}

School of Chemistry and Materials Science, iChEM, University of Science and Technology of China, Hefei 230026, China

\section{Fan Wu}

University of Science and Technology of China

Xiao-Kang Liu

University of Science and Technology of China

\section{Chunrong Ma}

University of Science and Technology of China

\section{Xiao Han}

University of Science and Technology of China

\section{Yihua Ran}

ShanghaiTech University

\section{Yan Zhang}

University of Science and Technology of China

\section{Zhiwen Zhang}

University of Science and Technology of China

\section{Guozhen Zhang}

University of Science and Technology of China

\section{Xiyu Li}

University of Science and Technology of China

\section{Jun Cai}

ShanghaiTech University

\section{Xun Hong}

University of Science and Technology of China https://orcid.org/0000-0003-2784-2868

\section{Zhi Liu}

School of Physical Science and Technology, Shanghai Tech University

\section{Jun Jiang}


Hefei National Laboratory for Physical Sciences at the Microscale, Collaborative Innovation Center of Chemistry for Energy Materials, CAS Center for Excellence in Nanoscience, School of Chemistry an https://orcid.org/0000-0002-6116-5605

\section{Tao Yao}

University of Science and Technology of China https://orcid.org/0000-0001-8699-8294

\section{Henny Bouwmeester}

Electrochemistry Research group, Membrane Science and Technology, MESA+ Institute for Nanotechnology, University of Twente, P.O. Box 217, 7500 AE, Enschede, The Netherlands https://orcid.org/0000-0003-0488-4141

\section{Physical Sciences - Article}

Keywords: catalysts, selective oxidation, methane

Posted Date: March 11th, 2021

DOl: https://doi.org/10.21203/rs.3.rs-265347/v1

License: (c) (i) This work is licensed under a Creative Commons Attribution 4.0 International License. Read Full License 


\section{Abstract}

Solid catalysts play vital roles as heterogeneous catalysts in numerous applications in the chemical, petrochemical, pharmaceutical, energy, food and automobile industries, facilitating large-scale production and facile product separation. However, conventional heterogeneous catalysts possess three major shortcomings that hinder their wide-spread application, including i) inferior catalytic activity and selectivity caused by a diversity of active sites, ii) tendency towards deactivation caused by agglomeration of catalyst particles, and iii) undesired carbon deposition in transformation of hydrocarbons. 1-4 In this research, we present a new kind of heterogeneous catalyst that consists of a liquid metal with dissolved catalytically active metal atoms, denoted as a heterogeneous droplet catalyst (HDC). Taking galinstan as the liquid metal with $\mathrm{Cu}$ as catalytically active metal solute (denoted as Cu$\mathrm{HDC}$ ) as an example, we demonstrate that the dissolved $\mathrm{Cu}$ atoms promote selective oxidation of methane into primary oxygenates at room temperature with a superb activity of $1940 \mathrm{mmol} \cdot \mathrm{gCu}-1 \cdot \mathrm{h}-1$ and a selectivity of over $90 \%$, outperforming state-of-the-art industrial catalysts. The liquid metal solvent ensures a high, entropically driven dispersion of the $\mathrm{Cu}$ atoms and prevents carbon deposition. The highly promising Cu-HDC catalyst shows a stable operation over 240 hours. By employing in-situ X-ray absorption spectroscopy (XAS), near ambient-pressure X-ray photoelectron spectroscopy (NAP-XPS) and density functional theory (DFT), we show that the narrow d-orbital of the dissolved $\mathrm{Cu}$ atoms in HDC facilitates formation of adsorbed methyl radicals ${ }^{*} \mathrm{CH} 3$ and impedes their transformation into ${ }^{*} \mathrm{CH} 2$ (where the * notation is used to denote an adsorption site) 5,6. These features enhance the selectivity towards primary oxygenates and prevent over-oxidization and formation of $\mathrm{C} 2+$ products. As the phase of the HDC is different from those of gas, aqueous and organic solution phases its separation is as facile as that of solid catalysts. Unlike conventional supported catalysts that are designed with well-defined crystal structures, crystal facets and defects, HDCs are characterized by weak interactions between the liquid metal support and the active metal solute, by having mobile surfaces and by dynamic catalytically active centers. These unprecedented features endow HDCs unique adsorption behavior and adaptive activation of molecules. For this reason, HDCs have the potential to become a major category of catalysts in the field of heterogeneous catalysis.

\section{Main Text}

Solid catalysts play vital roles as heterogeneous catalysts in numerous applications in the chemical, petrochemical, pharmaceutical, energy, food and automobile industries, facilitating large-scale production and facile product separation. However, conventional heterogeneous catalysts possess three major shortcomings that hinder their wide-spread application, including i) inferior catalytic activity and selectivity caused by a diversity of active sites, ii) tendency towards deactivation caused by agglomeration of catalyst particles, and iii) undesired carbon deposition in transformation of hydrocarbons. ${ }^{1-4}$ In this research, we present a new kind of heterogeneous catalyst that consists of a liquid metal with dissolved catalytically active metal atoms, denoted as a heterogeneous droplet catalyst (HDC). Taking galinstan as the liquid metal with $\mathrm{Cu}$ as catalytically active metal solute (denoted as Cu- 
$\mathrm{HDC}$ ) as an example, we demonstrate that the dissolved $\mathrm{Cu}$ atoms promote selective oxidation of methane into primary oxygenates at room temperature with a superb activity of $1940 \mathrm{mmol} \cdot \mathrm{g}_{\mathrm{Cu}}{ }^{-1} \cdot \mathrm{h}^{-1}$ and a selectivity of over $90 \%$, outperforming state-of-the-art industrial catalysts. The liquid metal solvent ensures a high, entropically driven dispersion of the $\mathrm{Cu}$ atoms and prevents carbon deposition. The highly promising Cu-HDC catalyst shows a stable operation over 240 hours. By employing in-situ X-ray absorption spectroscopy (XAS), near ambient-pressure X-ray photoelectron spectroscopy (NAP-XPS) and density functional theory (DFT), we show that the narrow $d$-orbital of the dissolved $\mathrm{Cu}$ atoms in HDC facilitates formation of adsorbed methyl radicals ${ }^{*} \mathrm{CH}_{3}$ and impedes their transformation into ${ }^{*} \mathrm{CH}_{2}$ (where the * notation is used to denote an adsorption site) ${ }^{5,6}$. These features enhance the selectivity towards primary oxygenates and prevent over-oxidization and formation of $\mathrm{C}_{2+}$ products. As the phase of the HDC is different from those of gas, aqueous and organic solution phases its separation is as facile as that of solid catalysts. Unlike conventional supported catalysts that are designed with well-defined crystal structures, crystal facets and defects, HDCs are characterized by weak interactions between the liquid metal support and the active metal solute, by having mobile surfaces and by dynamic catalytically active centers (Figure 1A). These unprecedented features endow HDCs unique adsorption behavior and adaptive activation of molecules. For this reason, HDCs have the potential to become a major category of catalysts in the field of heterogeneous catalysis.

Galinstan, a gallium-based eutectic alloy is a liquid at room temperature ${ }^{7-10}$. Different metals can be dissolved into galinstan, e.g., $\mathrm{Cu}, \mathrm{Ni}, \mathrm{Fe}, \mathrm{Co}, \mathrm{Zn}, \mathrm{Pt}, \mathrm{Pd}$, or $\mathrm{Au}$. The major challenge in order to facilitate practical application of HDCs as catalysts is to prevent their aggregation into larger droplets. To reduce the size of the droplets and to increase their specific surface area, we suspended the $\mathrm{Cu}-\mathrm{HDC}$ droplets in polydimethylsiloxane (PDMS) and solidified the obtained suspension by adding a cross-linking agent and subjecting it to an appropriate thermal treatment (Figure 1B). ${ }^{11,12}$ The cured PDMS rubber with embedded micro-sized Cu-HDC droplets in it (denoted as Cu-HDC@PDMS) can be used (see Supplementary Information: Preparation of M-HDC@PDMS).

The uniform distribution of $\mathrm{Cu}$ in the galinstan alloy was confirmed by energy dispersive X-ray spectroscopy (EDX). The obtained elemental mappings are shown in Figure 2A. The aberration-corrected scanning transmission electron microscope (STEM) images and associated 3D intensity profile demonstrate a disordered arrangement of the $\mathrm{Cu}$ atoms (Figure 2B, Figure $\mathrm{S} 1$ and Figure 2C). For a better observation, we extracted intensity values from the inverse fast Fourier transform (IFFT) image (Figure 2D) in six different directions. Every profile (Figure 2E) is found to exhibit aperiodicity in both amplitude and frequency, confirming the disordered nature of the $\mathrm{Cu}$ atoms. For a verification of the liquid characteristics of $\mathrm{Cu}-\mathrm{HDC}$, we employed cryogenic transmission electron microscopy (cryo-TEM). The ring-like selected-area electron diffraction (SAED) pattern recorded at room temperature confirms its amorphous nature (Figure 2F), while the SAED pattern recorded at $-196^{\circ} \mathrm{C}$, i.e., below the melting point of galinstan, shows bright spots indicative of a polycrystalline structure. Using X-Ray diffraction (XRD) it was found that galinstan is able to dissolve about $2 \mathrm{wt} \%$ of $\mathrm{Cu}$ at room temperature (Figure S2). Similar conclusions can be drawn by analyzing oscillations in extended X-ray absorption fine structure (EXAFS) 
spectra. Figure $2 \mathrm{G}$ compares EXAFS spectra of $\mathrm{Cu}$ foil, $\mathrm{Cu}-\mathrm{HDC}$ with $1 \mathrm{wt} \%$ and that with $2 \mathrm{wt} \% \mathrm{Cu}$, while Figure $2 \mathrm{H}$ shows corresponding wavelet transforms for the $\mathrm{k}^{3}$-weighted EXAFS signals (corresponding fitting results are shown in Figure S3 and Table S1, respectively). Fitting of the oscillations indicate that $\mathrm{Ga}$ atoms form the first coordination shell of $\mathrm{Cu}$ atoms in $\mathrm{Cu}-\mathrm{HDC}$, rather than In or Sn (having a higher Rfactor). The absence of second-nearest neighbors peaks above $4 \AA$ in the spectrum of Cu-HDC with 1 wt $\% \mathrm{Cu}$ indicates that agglomeration and crystallization did not occur. In contrast, these peaks emerge in the EXAFS spectrum of Cu-HDC with $2.2 \mathrm{wt} \%$ of $\mathrm{Cu}$.

Methane catalytic oxidation experiments using Cu-HDC@PDMS catalysts with different $\mathrm{Cu}$ contents were carried out in an autoclave with $\mathrm{H}_{2} \mathrm{O}_{2}$ as the oxidant at temperatures in the range $25-75^{\circ} \mathrm{C}^{13-16}$. The liquid and gas products were analyzed by ${ }^{1} \mathrm{H}$ nuclear magnetic resonance (NMR) and gas chromatography (GC), respectively. Corresponding data are shown in Figure 3A, revealing that generation of primary oxygenates, the most promising oxidation products of methane ${ }^{13,17}$, is dominant with a selectivity over $93 \%$ under all tested conditions. Control catalytic experiments were carried out using galinstan@PDMS (without Cu), Cu-HDC (without PDMS), pure PDMS and pure galinstan as catalysts, but none of these showed an activity and selectivity comparable to Cu-HDC@PDMS. A linear relationship between production rate and $\mathrm{Cu}$ content is found as shown in Figure $3 \mathrm{C}$, implying that $\mathrm{Cu}$-based moieties serve as catalytically active sites. As alluded to above, once the $\mathrm{Cu}$ content exceeds about $2 \mathrm{wt} \%$, a solid solution or alloy with crystalline $\mathrm{CuGa}_{2}$ appears as was confirmed by XRD and EXAFS. The formed $\mathrm{CuGa}_{2}$ species is less active in methane oxidation, which explains the departure from the linear relationship in Figure $3 \mathrm{C}$ at high $\mathrm{Cu}$ loadings. The production rates of undesired carbon dioxide and acetic acid are not significantly affected by the Cu content. The latter shows that the formation of these products is not associated with the presence of $\mathrm{Cu}$. Besides dispersing the liquid metal droplets, the PDMS rubber modulates the wettability and accessibility of the catalyst. We experienced that the liquid metal is quite wettable to water and methanol with contact angles of $8.6^{\circ}$ and close to $0^{\circ}$, respectively (Figure S4). This unfavorable wettability to water and methanol hinders catalytic sites from contact with gaseous methane and desorption of generated methanol. The use of PDMS modifies the wettability of the catalyst surface to become more aerophilic, which promotes the adsorption of methane and the desorption of methanol, thus enhancing the catalytic activity. It was found that Cu-HDC@PDMS shows a durability of at least 240 hours without any decline in catalytic performance. This outstanding performance originates mainly from two aspects: i) the solution characteristics of $\mathrm{Cu}-\mathrm{HDC}$ prevents aggregation of $\mathrm{Cu}$ atoms, and ii) its fluidity avoids carbon deposition, which was confirmed by EDX analysis of used catalysts (Figure S5 and Table S2). We further investigated the catalytic performance of Cu-HDC@PDMS at different temperatures and methane partial pressures. Corresponding results are shown in Figure 3D. As we identified Cu atoms as the catalytically active centers, the catalytic activity of Cu-HDC@PDMS was normalized to its Cu content. Even at room temperature and $1.5 \mathrm{MPa} \mathrm{CH}_{4}$, a catalytic performance as high as $1940 \mathrm{mmol} \cdot \mathrm{g}_{\mathrm{Cu}}{ }^{-}$ ${ }^{1} \cdot \mathrm{h}^{-1}$ (with a turnover frequency (TOF) of $124.1 \mathrm{~mol} \cdot \mathrm{mol}_{\mathrm{Cu}^{-1}} \cdot \mathrm{h}^{-1}$ ) can be achieved. According to the best of our knowledge, this is the highest production rate of primary oxygenates from methane reported to date (cf. Table S3). Figure S6 shows that the reaction is first order in methane. A value of $31.3 \pm 1.7 \mathrm{~kJ} \mathrm{~mol}{ }^{-1}$ 
was obtained for the apparent activation energy (Figure S7). This value is lower than reported for other catalysts and underscores the superior catalytic activity exhibited by Cu-HDC@PDMS ${ }^{13,18-20}$. Figure S8 confirms a linear relationship between the amount of oxygenates formed and the time of reaction. Within the duration of the experiment the catalyst does not show any sign of deactivation. Analysis by ${ }^{13} \mathrm{C}$ and ${ }^{1} \mathrm{H}$ nuclear magnetic resonance (NMR) showed that products $\left(\mathrm{CH}_{3} \mathrm{OOH}\right.$ and $\left.\mathrm{HOCH}_{2} \mathrm{OOH}\right)$ are only observed when ${ }^{13} \mathrm{CH}_{4}$ is used as reactant, confirming that methane is the only source of carbon in the products (Figure S9) ${ }^{17}$.

The remarkable catalytic performance of Cu-HDC prompted us to investigate the underlying mechanism of the catalytic reaction. In-situ XAFS was used to study the local chemical environment of the Cu atoms during reaction. Measurements were performed using a home-made gas-tight reaction chamber equipped with a vitreous carbon window which allowed us to obtain the EXAFS signal (Figure S10). Spectra were collected of $\mathrm{Cu}-\mathrm{HDC}$ in pristine form, immersed in $30 \%$ hydrogen peroxide and under reaction conditions (Figures $3 \mathrm{~F}-3 \mathrm{H}$ ). Curve fitting of the spectrum obtained for pristine $\mathrm{Cu}-\mathrm{HDC}$ only reveals the $\mathrm{Cu}-\mathrm{Ga}$ bond. A peak at ca. $1.4 \AA$ appears in the spectrum of Cu-HDC immersed in $30 \%$ hydrogen peroxide. Curve fitting shows that this peak corresponds to $\mathrm{Cu}$ atoms bonded to a single $\mathrm{O}$ atom at a distance of about $1.9 \AA$. The EXAFS spectrum recorded under $2.0 \mathrm{MPa} \mathrm{CH}_{4}$ reveals that the $\mathrm{Cu}-\mathrm{O}$ peak is shifting to $1.5 \AA$, corresponding to a slight increase of the $\mathrm{Cu}-\mathrm{O}$ interatomic distance to about $2.0 \AA$. Exposure to $\mathrm{CH}_{4}$ leads to formation of $\mathrm{Cu}-\mathrm{O}-\mathrm{CH}_{3}$ and, hence, weakening of the $\mathrm{Cu}-\mathrm{O}$ bond ${ }^{21}$. To verify these findings, we performed ambient-pressure X-ray photoelectron spectroscopy (APXPS) (Figure 3I, Figure S11). The $\mathrm{C}_{1 \mathrm{~s}}$ spectra recorded under $0.5 \mathrm{mbar} \mathrm{CH}_{4}$ reveal the existence of $\mathrm{CH}_{\mathrm{x}}(285.6 \mathrm{eV})$ and molecular $\mathrm{CH}_{4}(287.0$ $\mathrm{eV})$. New peaks appear when adding $0.5 \mathrm{mbar}$ of $\mathrm{O}_{2}$ due to the formation of $\mathrm{OCH}_{3}(286.5 \mathrm{eV})$ and $\mathrm{CO}_{\mathrm{x}}$ (289.8 eV). The $\mathrm{C}_{1 \mathrm{~s}}$ peak corresponding to $\mathrm{OCH}_{3}$ is found to dominate in a mixed $\mathrm{CH}_{4} / \mathrm{O}_{2}$ atmosphere, suggesting that $\mathrm{OCH}_{3}$ is a stable intermediate in methane oxidation (Figure $3 \mathrm{~J}$ ) 22,23 . The data further confirms the high selectivity of the $\mathrm{Cu}-\mathrm{HDC}$ catalyst as the relative intensity of the $\mathrm{CO}_{\mathrm{x}}$ peak is found very small (10\%).

First-principle DFT calculations were performed to investigate the high catalytic activity of Cu-HDC droplets towards oxygenates. As expected, the computations disclose a low energy barrier for migration of $\mathrm{Cu}$ atoms from the interior of the droplets to their surface, suggesting a high possibility for formation of Cu-centered active sites for the catalytic surface reaction (Figure S12). These calculations were performed assuming the liquid metal to consist of pure gallium (rather than a mixture of gallium, indium and tin). We further calculated the reaction pathways for partial oxidation of $\mathrm{CH}_{4}$ on the surfaces of $\mathrm{Cu}$ $\mathrm{HDC}$, pure gallium and on $\mathrm{Cu}(111)$ as shown in Figures S13, S14 and S15, respectively. In these figures, Roman numbering is used to designate sequential stages in the mechanism. The calculations reveal that surface $\mathrm{Cu}$ and two adjacent $\mathrm{Ga}$ atoms abstract one $\mathrm{O}$ atom from $\mathrm{H}_{2} \mathrm{O}_{2}$ (II), forming a Cu-O- $\mathrm{Ga}_{2}$ motif on the surface of Cu-HDC (III), acting as the site for $\mathrm{CH}_{4}$ adsorption (Figure S13). A bond distance of $1.88 \AA$ is calculated for the $\mathrm{Cu}-\mathrm{O}$ bond, which is consistent with the value determined by in-situ XAFS. Upon adsorption of $\mathrm{CH}_{4}$ (III), one of its $\mathrm{H}$ atoms is abstracted by the ${ }^{*} \mathrm{O}$ atom, whereby the ${ }^{*} \mathrm{CH}_{3}$ methyl radical 
is formed (IV). This is the decisive step in the overall reaction with an energy barrier (TS1) of $1.07 \mathrm{eV}$ (by comparison with $1.38 \mathrm{eV}$ (Figure S11) and $1.56 \mathrm{eV}$ (Figure S12) calculated for similar reaction steps occurring on pure gallium and $\mathrm{Cu}(111)$, respectively). Two reaction steps may follow: $\left(\mathrm{V}_{\mathrm{a}}\right){ }^{*} \mathrm{CH}_{3}$ reacts with $\mathrm{H}_{2} \mathrm{O}_{2}$ to form $\mathrm{CH}_{3} \mathrm{OH}$, or $\left(\mathrm{V}_{\mathrm{b}}\right){ }^{*} \mathrm{CH}_{3}$ is further dehydrogenated to ${ }^{*} \mathrm{CH}_{2}$. The ${ }^{*} \mathrm{CH}_{2}$ radical is the precursor intermediate of oxidation of carbon to higher oxidation states. The calculated energy barrier for reaction step $\left(\mathrm{V}_{\mathrm{b}}\right)$ is $1.36 \mathrm{eV}$ (TS2), which is high and implies that oxidation of $\mathrm{CH}_{4}$ on Cu-HDC would not proceed any further. It may be noted that the energy barrier for this reaction occurring on $\mathrm{Cu}(111)$ is found to be only $1.03 \mathrm{eV}$ (Figure S12). The obtained results clearly demonstrate that reaction via reaction step $\left(\mathrm{V}_{\mathrm{a}}\right)$ is the more favorable pathway to produce $\mathrm{CH}_{3} \mathrm{OH}(\mathrm{VI})$ on Cu-HDC. The formed $\mathrm{CH}_{3} \mathrm{OH}$ may be adsorbed on the $\mathrm{Cu}$ atom (VII). Subsequent $\mathrm{H}$-abstraction (VIII) and reaction of the formed intermediate * $\mathrm{OCH}_{3}$ with $\mathrm{H}_{2} \mathrm{O}_{2}(\mathrm{IX})$ will generate $\mathrm{CH}_{3} \mathrm{OOH}(\mathrm{X})$.

To understand the superior activity and selectivity of $\mathrm{Cu}-\mathrm{HDC}$ over $\mathrm{Cu}(111)$ in methane oxidation, a comparative computational analysis of the electronic structures of the TS1 transition states was carried out. In both cases, the ${ }^{*} \mathrm{CH}_{3}$ radical resides on top of the $\mathrm{Cu}$ atoms, denoted as $\mathrm{Cu}$-HDC_TS1 (Figure S13) and $\mathrm{Cu}(111)$ _TS1 (Figure S15), respectively. As shown in Figure 4, the orbital interaction between ${ }^{*} \mathrm{CH}_{3}$ and $\mathrm{Cu}-\mathrm{HDC}$ is substantially stronger than that between ${ }^{*} \mathrm{CH}_{3}$ and $\mathrm{Cu}(111)$, which leads to a substantial lower energy barrier for $\mathrm{CH}_{4}$ activation on $\mathrm{Cu}-\mathrm{HDC}$ by comparison with that on $\mathrm{Cu}(111)$. The projected density of states (pDOS) of the $\mathrm{Cu} 3 d$ orbitals in Cu-HDC (Figure 4A) exhibits as a narrow peak, suggesting a very weak coupling between the $\mathrm{Cu} 3 d$ orbitals and the surrounding $\mathrm{Ga}$ orbitals. In contrast, the $3 d$ orbitals in $\mathrm{Cu}(111)$ are split and broadly distributed (Figure 4B). The difference in the $\mathrm{Cu} 3 d$ orbital distributions accounts for the different orbital interactions between $\mathrm{Cu}$ and ${ }^{*} \mathrm{CH}_{3}$ in the respective TS1 transition states. The $\mathrm{Cu} 3 d$ and ${ }^{*} \mathrm{CH}_{3}$ non-bonding (nb) orbitals are strongly coupled in Cu-HDC_TS1, but hardly interact with each other in $\mathrm{Cu}(111)_{-} \mathrm{TS} 1$. As a result, a $\mathrm{Cu}-\mathrm{C}$ bond is formed when ${ }^{*} \mathrm{CH}_{3}$ is adsorbed onto $\mathrm{CuGa}_{125}$, while ${ }^{*} \mathrm{CH}_{3}$ almost retains its radical character when adsorbed onto the $\mathrm{Cu}(111)$ surface. These results are strengthened by the projected crystal orbital Hamilton population (pCOHP) calculations (Table S4), which reveal a Cu-C bond strength of +0.24 lel for adsorption of ${ }^{*} \mathrm{CH}_{3}$ onto $\mathrm{Cu}-\mathrm{HDC}$ and +0.03 lel in the case of adsorption onto $\mathrm{Cu}(111)$. Moreover, the differential charge density profiles indicate that there is an appreciably higher charge density between the $\mathrm{Cu}$ and $\mathrm{C}$ atoms in Cu-HDC_TS1 (Figure 4C) than in $\mathrm{Cu}(111) \_\mathrm{TS} 1$ (Figure 4D). The ${ }^{*} \mathrm{CH}_{3}$ radical resides directly atop of the $\mathrm{Cu}$ atom on the $\mathrm{Cu}-\mathrm{HDC}$ surface, with a binding energy of $-1.21 \mathrm{eV}$ (Figure 4E), while it moves to a hollow site on the $\mathrm{Cu}(111)$ surface, with a much larger binding energy of $-1.81 \mathrm{eV}$ (Figure 4F). Such a big difference in the binding strength can be explained in two alternative ways. One, ${ }^{*} \mathrm{CH}_{3}$ forms three $\mathrm{Cu}-\mathrm{C}$ bonds of length $2.23 \AA$ when it adsorbs at the hollow site on $\mathrm{Cu}(111)$, while there is only one $\mathrm{Cu}-\mathrm{C}$ bond of length $2.02 \AA$ when it adsorbs atop a $\mathrm{Cu}$ atom on the $\mathrm{Cu}-\mathrm{HDC}$ surface. Albeit the individual $\mathrm{Cu}-\mathrm{C}$ bond is weaker in $\mathrm{CH}_{3}-\mathrm{Cu}(111)$ than in $\mathrm{CH}_{3}-\mathrm{Cu}-\mathrm{HDC}$, two more $\mathrm{Cu}-\mathrm{C}$ bonds are formed in the former complex resulting in an overall stronger binding strength. Another explanation is that the $d-\pi$ orbital hybridization between $\mathrm{Cu}$ and $\mathrm{C}$ atoms contributing to the $\mathrm{Cu}-\mathrm{C}$ bonding is appreciably stabilized in the $\mathrm{Cu}(111)_{-}{ }^{*} \mathrm{CH}_{3}$ complex, while it is barely changed in $\mathrm{Cu}-\mathrm{HDC}_{-}{ }^{*} \mathrm{CH}_{3}$, as shown in Figure $4 \mathrm{~A}$ and $4 \mathrm{~B}$, respectively. The much stronger binding 
of ${ }^{*} \mathrm{CH}_{3}$ on the $\mathrm{Cu}(111)$ surface is further confirmed by the pCOHP analysis (Table S4), showing a larger $d-p$ population (integral of $-\mathrm{pCOHP}$ ) on the $\mathrm{Cu}-\mathrm{C}$ bond $\left(+0.73\right.$ le| for $\mathrm{Cu}(111)_{-}{ }^{*} \mathrm{CH}_{3}$ vs. +0.62 lel for $\mathrm{Cu}-$ $\mathrm{HDC}_{-}{ }^{*} \mathrm{CH}_{3}$ ). On the basis of above analysis it is concluded that, because of the narrow $3 d$ orbital distribution, activation of $\mathrm{CH}_{4}$ is more favorable on $\mathrm{Cu}-\mathrm{HDC}$, while the occurrence of over-oxidation and formation of $\mathrm{C}_{2+}$ products is less likely than on the $\mathrm{Cu}(111)$ surface. Hence, $\mathrm{Cu}-\mathrm{HDC}$ outperforms $\mathrm{Cu}(111)$ in terms of both activity and selectivity of methane oxidation.

In conclusion, in this research we have demonstrated that a liquid metal with a catalytically active metal solute can act as a highly efficient and durable heterogeneous catalyst. By employing a liquid metal with dissolved $\mathrm{Cu}$, we achieved a state-of-the-art catalytic performance in the selective oxidation of methane, with an activity of $1940 \mathrm{mmol} \cdot \mathrm{g}_{\mathrm{Cu}}{ }^{-1} \cdot \mathrm{h}^{-1}$ and a selectivity over $90 \%$ at room temperature. In-situ XAFS and NAP-

XPS experiments and DFT calculations reveal that the active $\mathrm{Cu}$ atoms present at the surface of the liquid metal droplet facilitates adsorption of methane, generates methyl radicals and prevents over-oxidation and formation of $\mathrm{C}_{2+}$ products. We believe that our findings offer new opportunities and exciting challenges for discovery of novel heterogeneous catalysts.

\section{Declarations}

\section{Acknowledgement}

This work was financially supported by the National Key R\&D Program of China 2017YFA (0208300 and 0700104), the National Natural Science Foundation of China (21671180), the CAS Fujian Institute of Innovation and the DNL Cooperation Fund (DNL201918). The authors also acknowledge the photoemission end stations BL1W1B in the Beijing Synchrotron Radiation Facility (BSRF), BL14W1 in Shanghai Synchrotron Radiation Facility (SSRF), BL10B and BL11U in the National Synchrotron Radiation Laboratory (NSRL) for providing assistance in characterization. Part of this research was carried out using the SPECS NAP-XPS instrument, being supported by the ME2 project under contract no. 11227902 from the National Natural Science Foundation of China. The numerical calculations presented in this work were performed on the supercomputer system in the Supercomputing Center of University of Science and Technology of China.

\section{Author contributions}

Y.W. conceived and designed the experiments. H.Z., X.W., C.M., Y.Z and Z.Z. performed the synthesis of catalysts and catalytic performance. X.H. and H.X. helped with the spherical aberration electron microscopy tests and discuss. H.Z., X.W., X.L. and T.Y. designed and performed XAFS experiments. H.Z., C.M., Y.R., J.C. and Z.L. designed and performed NAPXPS experiments. F.W., G.Z., X.L. and J.J. performed DFT calculations and analyzed results. H.Z., X.W., H.J.M.B and Y.W. wrote the manuscript. All of the 
authors discussed the results and commented on the manuscript. H.Z., X.W., F.W. and X.L. contributed to this work equally.

\section{Competing interests}

The authors declare no competing interests.

\section{References}

$1 \quad$ Hutchings, G. J. et al. Modern developments in catalysis. (World Scientific, 2016).

2 Mizuno, N. \& Misono, M. Heterogeneous catalysis. Chem. Rev. 98, 199-218 (1998).

3 Bell, A. T. The impact of nanoscience on heterogeneous catalysis. Science 299, 1688-1691 (2003).

4 Thomas, J. M. \& Thomas, W. J. Principles and practice of heterogeneous catalysis. (John Wiley \& Sons, 2014).

5 Greiner, M. T. et al. Free-atom-like d states in single-atom alloy catalysts. Nat. Chem. 10, 1008-1015 (2018).

6 Taccardi, N. et al. Gallium-rich Pd-Ga phases as supported liquid metal catalysts. Nat. Chem. 9, 862-867 (2017).

7 Daeneke, T. et al. Liquid metals: fundamentals and applications in chemistry. Chem. Soc. Rev. 47, 4073-4111 (2018).

8 Hoshyargar, F., Crawford, J. \& O'Mullane, A. P. Galvanic replacement of the liquid metal galinstan. J. Am. Chem. Soc. 139, 1464-1471 (2017).

9 Wang, Q., Yu, Y. \& Liu, J. Preparations, characteristics and applications of the functional liquid metal materials. Adv. Eng. Mater. 20, 1700781 (2018).

10 Yan, J. et al. Shape-controlled synthesis of liquid metal nanodroplets for photothermal therapy. Nano. Res. 12, 1313-1320 (2019).

11 Fassler, A. \& Majidi, C. Liquid-phase metal inclusions for a conductive polymer composite. Adv. Mater. 27, 1928-1932 (2015).

12 Markvicka, E. J., Bartlett, M. D., Huang, X. \& Majidi, C. An autonomously electrically self-healing liquid metal-elastomer composite for robust soft-matter robotics and electronics. Nat. Mater. 17, 618-624 (2018).

13 Meng, X. et al. Direct Methane Conversion under Mild Condition by Thermo-, Electro-, or Photocatalysis. Chem 5, 2296-2325 (2019). 
14 Shan, J., Li, M., Allard, L. F., Lee, S. \& Flytzani-Stephanopoulos, M. Mild oxidation of methane to methanol or acetic acid on supported isolated rhodium catalysts. Nature 551, 605-608 (2017).

15 Lin, J. \& Wang, X. Rh single atom catalyst for direct conversion of methane to oxygenates. Sci. China Mater 61, 758-760 (2018).

16 Yang, W. et al. First principles study on methane reforming over Ni/TiO 2 (110) surface in solid oxide fuel cells under dry and wet atmospheres. Sci. China Mater 63, 364-374 (2020).

17 Cui, X. et al. Room-Temperature Methane Conversion by Graphene-Confined Single Iron Atoms. Chem 4, 1902-1910, (2018).

18 Ravi, M. et al. Misconceptions and challenges in methane-to-methanol over transition-metalexchanged zeolites. Nat. Catal. 2, 485-494 (2019).

19 Tomkins, P., Ranocchiari, M. \& van Bokhoven, J. A. Direct conversion of methane to methanol under mild conditions over Cu-zeolites and beyond. Acc. Chem. Res. 50, 418-425 (2017).

20 Schwach, P., Pan, X. \& Bao, X. Direct conversion of methane to value-added chemicals over heterogeneous catalysts: challenges and prospects. Chem. Rev. 117, 8497-8520 (2017).

21 Ikuno, T. et al. Methane oxidation to methanol catalyzed by Cu-oxo clusters stabilized in NU-1000 metal-organic framework. J. Am. Chem. Soc. 139, 10294-10301 (2017).

22 Liu, Z. et al. Water-promoted interfacial pathways in methane oxidation to methanol on a CeO2Cu20 catalyst. Science $368,513-517$ (2020).

23 Lustemberg, P. G. et al. Direct conversion of methane to methanol on Ni-Ceria surfaces: metalsupport interactions and water-enabled catalytic conversion by site blocking. J. Am. Chem. Soc. 140, 7681-7687 (2018).

\section{Figures}


A (i)
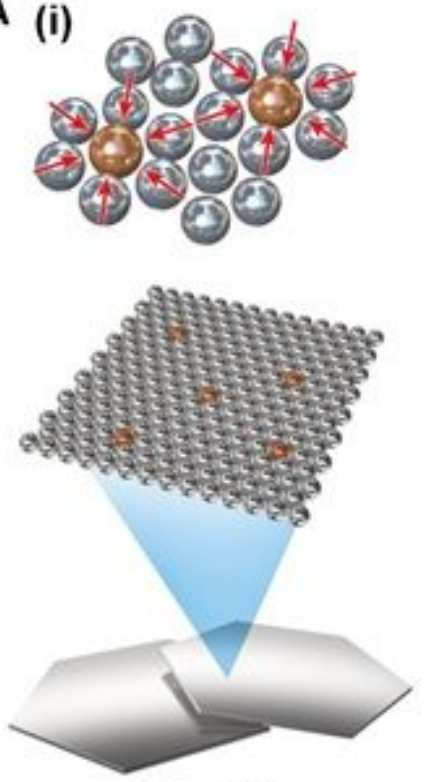

Crystalline (ii)
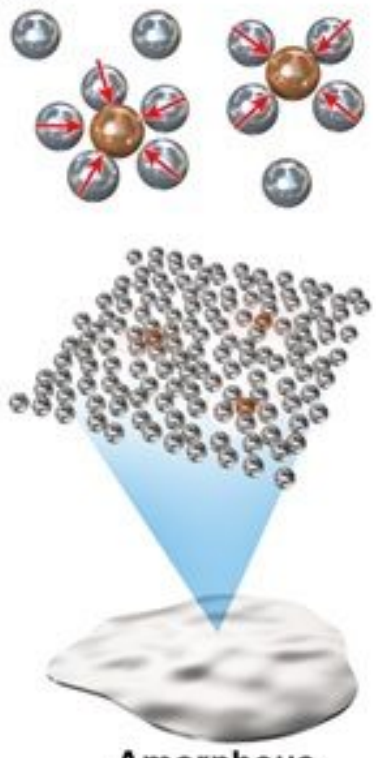

Amorphous (iii)
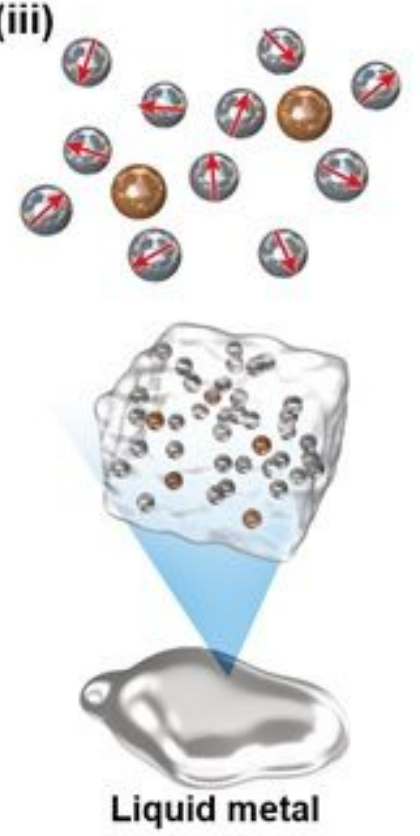

B

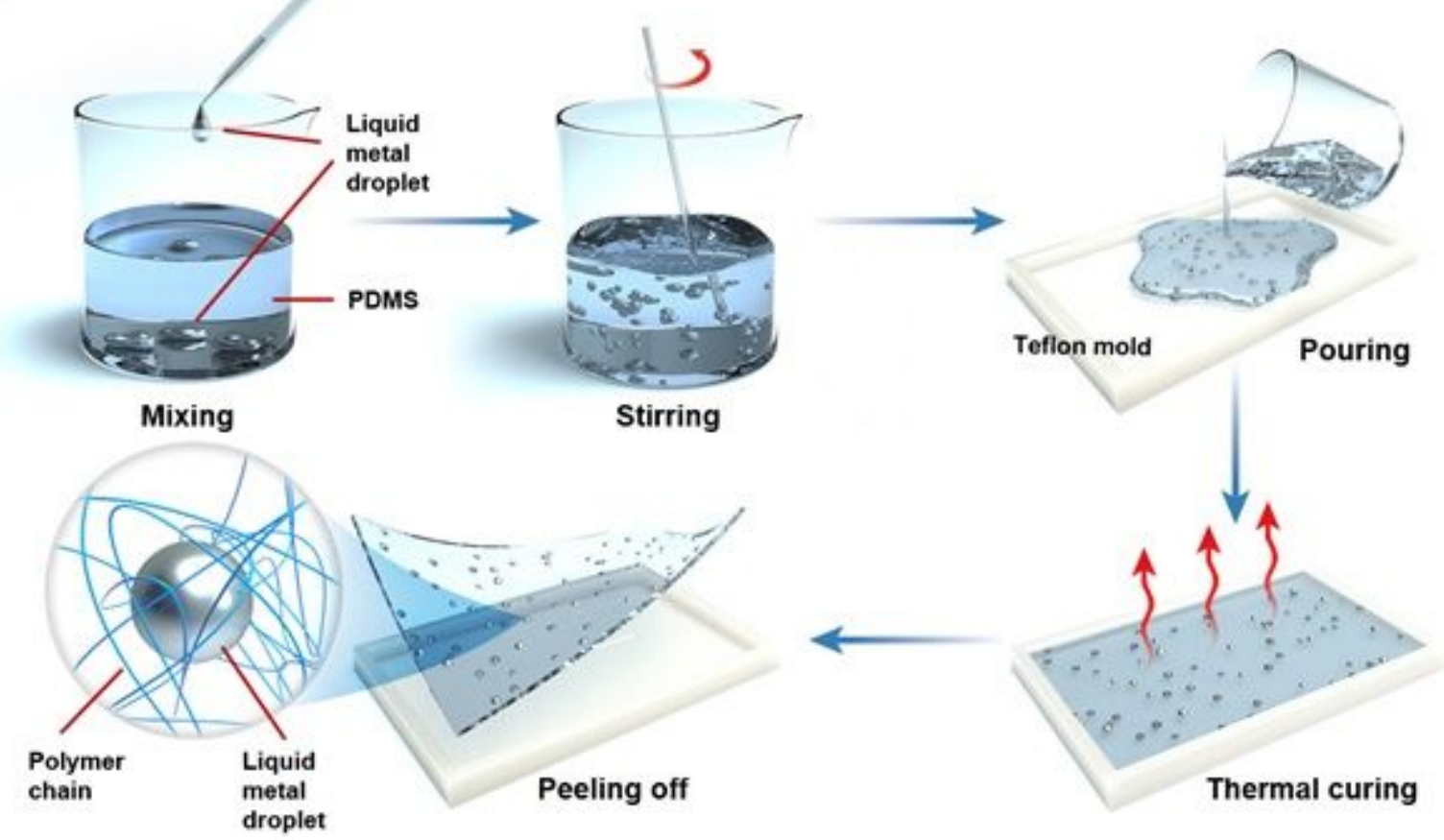

\section{Figure 1}

(A) Different type of catalysts: (i) crystalline, (ii) amorphous, with a random atomic arrangement, and (iii) liquid metal, with a dynamic atomic arrangement. (B) Schematics of preparation of M-HDC@PDMS. 

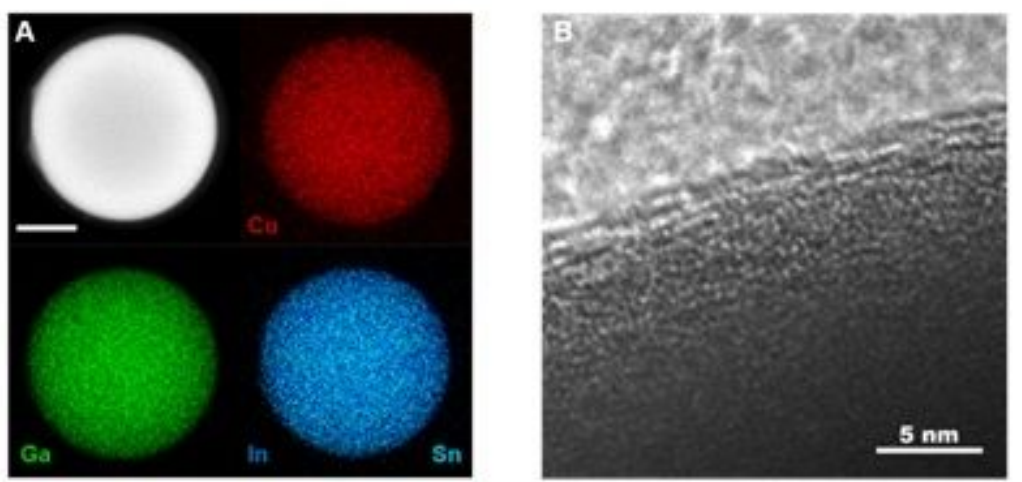

c
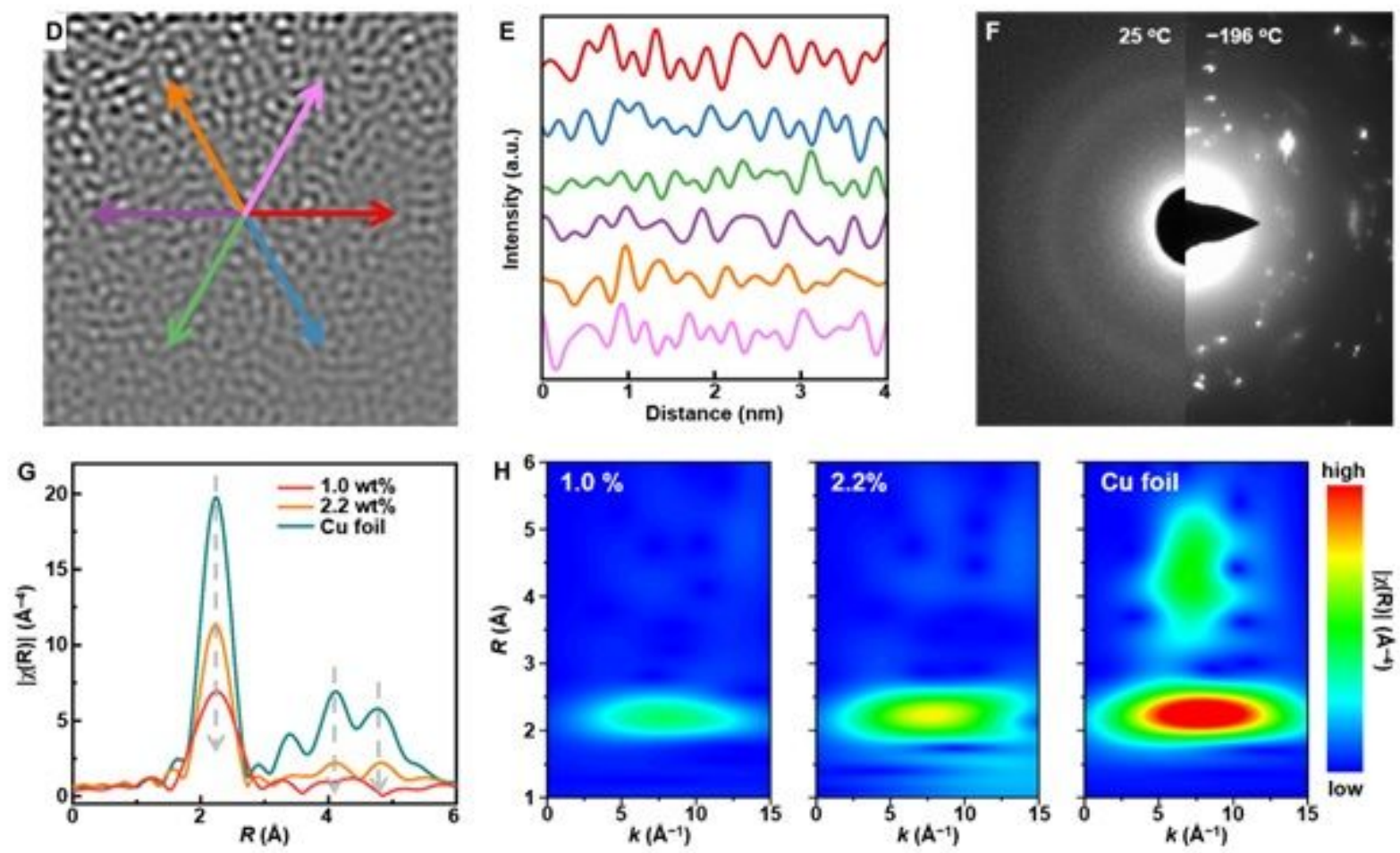

\section{Figure 2}

Characterization of Cu-HDC. (A) EDX elemental mapping analysis. The scale bar represents $50 \mathrm{~nm}$. (B) HAADF-STEM image and (C) corresponding 3D intensity profile image. (D) IFFT image of (B). (E) intensity profiles along different directions as denoted in (D). (F) SAED patterns recorded at 25 oC and -196 oC. (G) Cu K-edge EXAFS spectra in R-space recorded for Cu-HDC with a Cu loading of $1.0 \mathrm{wt} \%$ and $2.2 \mathrm{wt} \%$, and $\mathrm{Cu}$ foil, and $\mathrm{H}$ ) corresponding wavelet transforms for the k3-weighted EXAFS signals. 


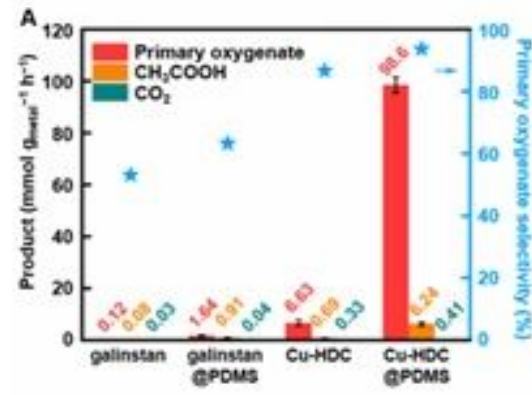

D
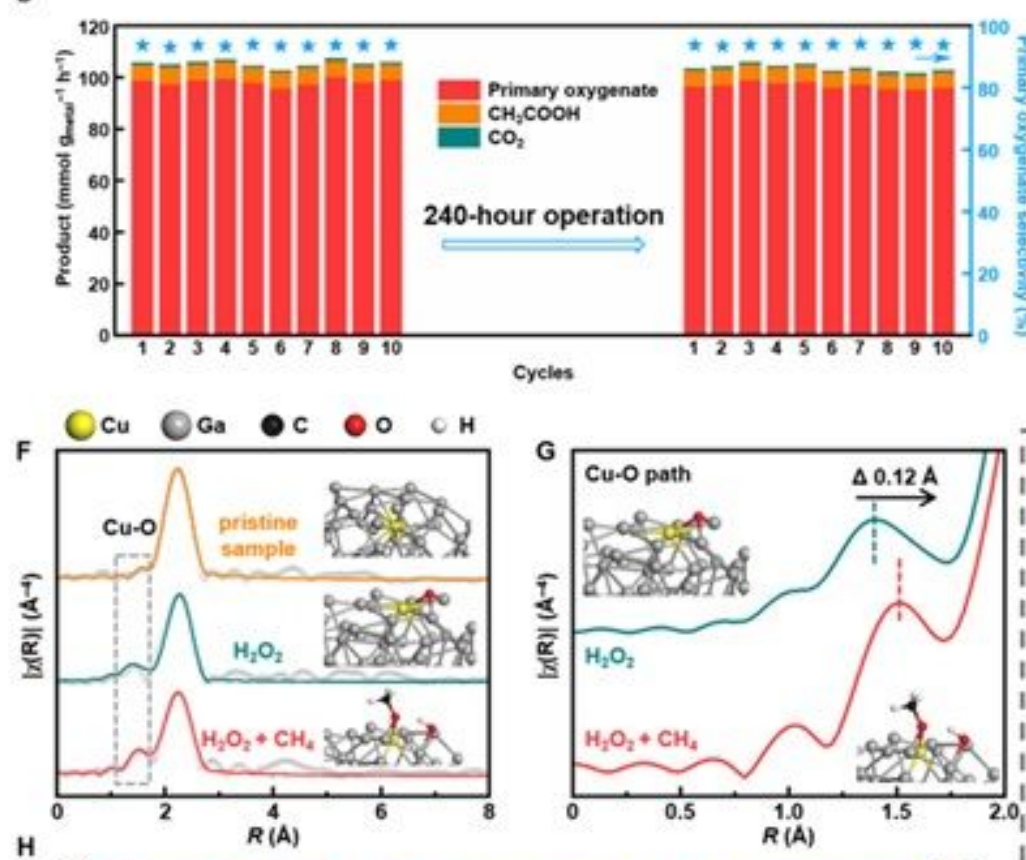

$\mathrm{H}$
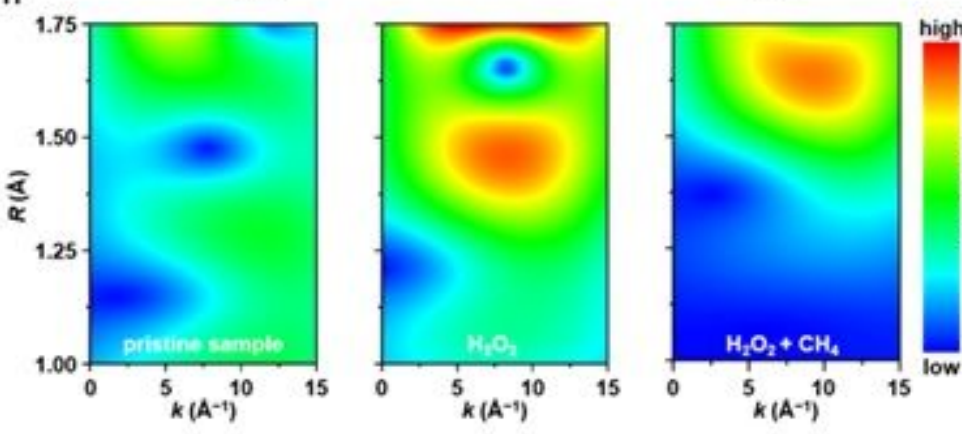
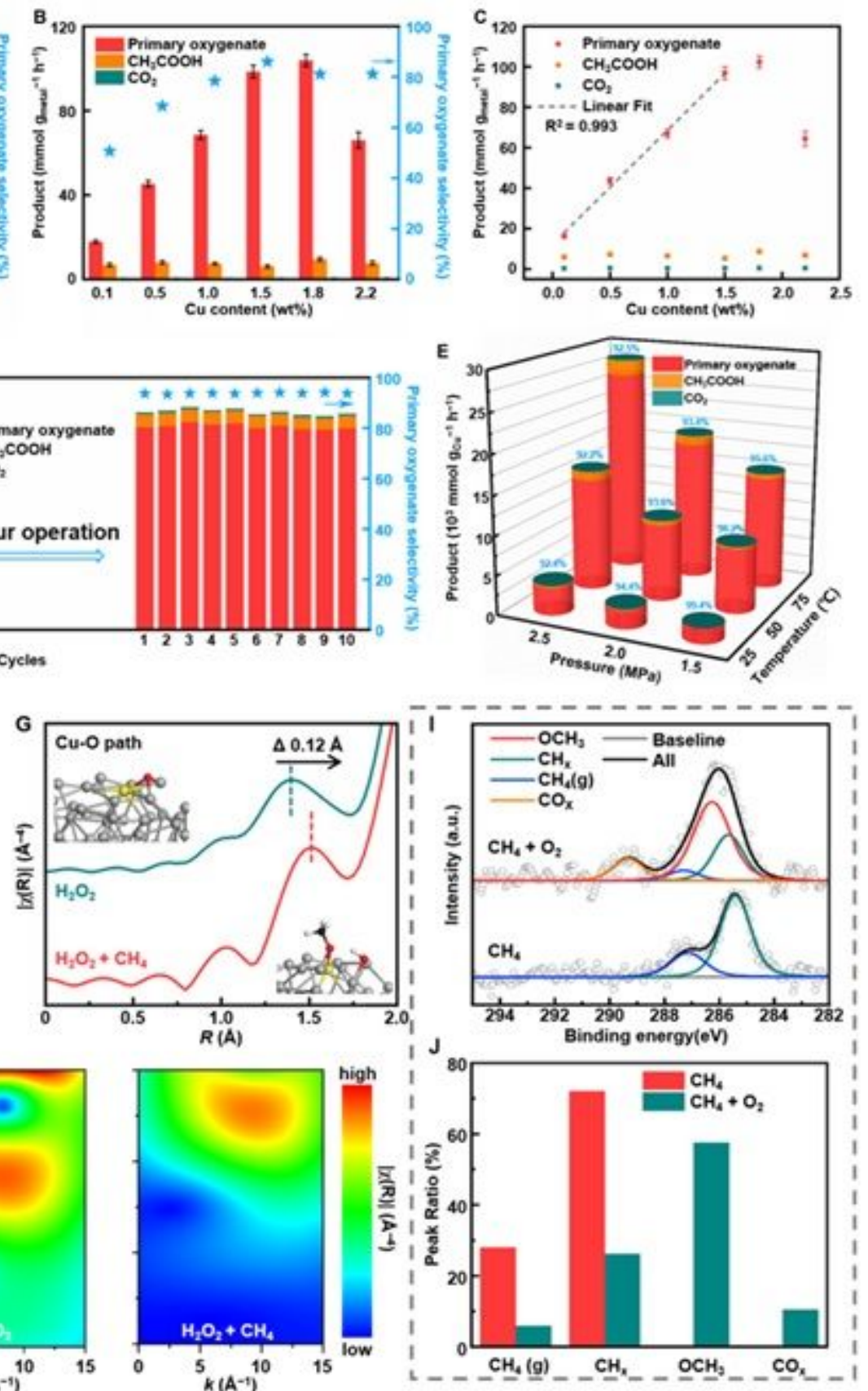

\section{Figure 3}

Catalytic performance in methane oxidation. (A) Production rate and selectivity towards primary oxygenates for galinstan, galinstan@PDMS, Cu-HDC (1 wt\% Cu) and Cu-HDC@PDMS (1 wt\% Cu in CuHDC). (B) Production rate and selectivity towards primary oxygenates for Cu-HDC@PDMS with different Cu contents. (C) Correlation between production rate and Cu content for Cu-HDC@PDMS. (D) Long-term stability Cu-HDC@PDMS. (E) Production rate (normalized to Cu content) and selectivity towards primary oxygenates at different temperatures and methane partial pressures. $(F)$ and $(G)$ Cu K-edge EXAFS spectra in R space recorded for pristine $\mathrm{Cu}-\mathrm{HDC}$, Cu-HDC immersed in $\mathrm{H} 2 \mathrm{O} 2$, and $\mathrm{Cu}-\mathrm{HDC}$ under reaction 
conditions, and $(\mathrm{H})$ corresponding wavelet transforms for the k3-weighted EXAFS signals. (I) APXPS spectra for $\mathrm{Cu}-\mathrm{HDC}$ recorded under $\mathrm{CH} 4$ and $\mathrm{CH} 4 / \mathrm{O} 2$ atmospheres. (J) Peak ratio of signals in (I).

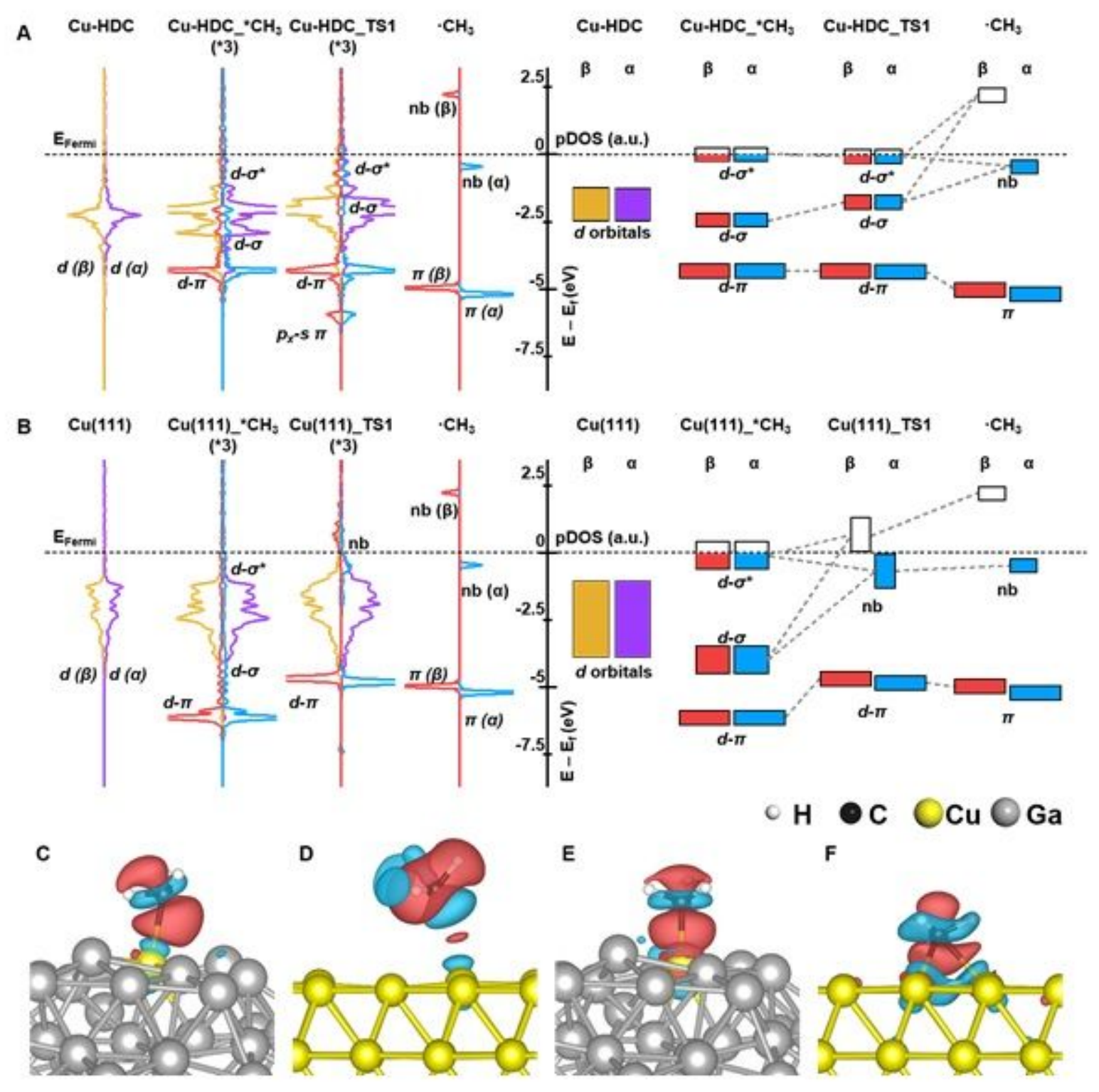

\section{Figure 4}

Bonding analysis of key reaction complexes during methane oxidation on $\mathrm{Cu}-\mathrm{HDC}$ and $\mathrm{Cu}(111)$ based on DFT calculations. Top and middle panels display the projected density of states (pDOS) and molecular orbitals of $\bullet \mathrm{CH} 3$ radical, $\mathrm{Cu} 3 \mathrm{~d}$ orbital on Cu-HDC, Cu-HDC_TS1 (magnified 3 times) and $\mathrm{Cu}-\mathrm{HDC} \_{ }_{-} \mathrm{CH} 3$ (magnified 3 times), $\mathrm{Cu} 3 \mathrm{~d}$ orbital on $\mathrm{Cu}(111), \mathrm{Cu}(111) \_$TS1 (magnified 3 times) and $\mathrm{Cu}(111) \_{ }^{*} \mathrm{CH} 3$ (magnified 3 times). $a$ and $\beta$ refer to spin-up and spin-down states. The bottom panel displays the charge density difference of ${ }^{*} \mathrm{CH} 3$ on $\mathrm{Cu}-\mathrm{HDC}$ and $\mathrm{Cu}(111)$ with red and blue regions indicating charge accumulation and depletion, respectively: (C) Cu-HDC_TS1, (D) $\mathrm{Cu}(111) \_T S 1$, (E) Cu-HDC_${ }^{*} \mathrm{CH} 3$ and (F) $\mathrm{Cu}(111) \_\mathrm{CH} 3$.

\section{Supplementary Files}


This is a list of supplementary files associated with this preprint. Click to download.

- HeterogeneousdropletcatalystforselectiveoxidationofmethaneSupplementaryinformation.docx 